\title{
Ionospheric influence on the seismo-telluric current related to electromagnetic signals observed before the Wenchuan $M_{S} 8.0$ earthquake
}

\author{
Mei Li ${ }^{1,2}$, Handong $\mathrm{Tan}^{2}$, and Meng Cao ${ }^{2}$ \\ ${ }^{1}$ China Earthquake Networks Center, China Earthquake Administration, No. 5, Sanlihe Nanheng Street, \\ Xicheng District, 100045 Beijing, China \\ ${ }^{2}$ China University of Geosciences, No. 29, Xueyuan Road, Haidian District, 100083 Beijing, China \\ Correspondence to: Mei Li (limeixuxl@seis.ac.cn)
}

Received: 8 June 2016 - Published in Solid Earth Discuss.: 30 June 2016

Revised: 12 September 2016 - Accepted: 13 September 2016 - Published: 19 October 2016

\begin{abstract}
A three-layer (Earth-air-ionosphere) physical model, as well as a two-layer (Earth-air) model, is employed in this paper to investigate the ionospheric effect on the wave fields for a finite length dipole current source co-located at a hypocenter depth and along the main fault of an earthquake when the distance between the epicenter and an observing station is up to $1000 \mathrm{~km}$ or even more. The results show that all electrical fields are free of ionospheric effects for different frequencies in a relative short range, e.g., $\sim 300 \mathrm{~km}$ for $f=1 \mathrm{~Hz}$, implying the ionospheric influence on electromagnetic fields can be neglected within this range, which becomes smaller as the frequency increases. However, the ionosphere can give a constructive interference to the waves passing through and make them decay slowly when an observation is out of this range; moreover, the ionospheric effect can be up to 1-2 orders of magnitude of the electrical fields. For a ground-based observable $1.3 \mathrm{mV} \mathrm{m}^{-1}$ electric signal at $f=1 \mathrm{~Hz} 1440 \mathrm{~km}$ away from the Wenchuan $M_{S} 8.0$ earthquake, the expected seismo-telluric current magnitude for the Earth-air-ionosphere model is of $5.0 \times 10^{7} \mathrm{~A}, 1 \mathrm{mag}$ nitude smaller than the current value of $3.7 \times 10^{8} \mathrm{~A}$ obtained by the Earth-air model free of ionospheric effects. This indicates that the ionosphere facilitates the electromagnetic wave propagation, as if the detectability of the system were improved effectively and it is easier to record a signal even for stations located at distances beyond their detectability thresholds. Furthermore, the radiating patterns of the electrical field components $\left|E_{x}\right|$ and $\left|E_{y}\right|$ are complementary to each other, although any two-dimensional (2-D) power distribution of
\end{abstract}

these components shows strong power areas as well as weak ones, which is advantageous to register a signal if the observing system is designed to measure both of them instead of only one.

\section{Introduction}

The fact that electromagnetic (EM) emissions accompany every stage of some large earthquake preparations seems undebatable, although short-term earthquake prediction is still one of the most challenging targets in Earth science today (Eftaxias et al., 2003). Meanwhile, the ultra low frequency (ULF) band is of particular interest because only EM signals in the ULF range and at lower frequencies that originated in the Earth's crust can be easily recorded at the Earth's surface without significant attenuation, compared with "high" frequency emissions that might be emitted at epicenter depths at more than $10 \mathrm{~km}$, even several hundreds of kilometers. Recently, an increasing number of ground-based observing ULF electromagnetic emissions related to strong earthquakes have been recorded at a distance of several, hundreds, and even several thousands of kilometers. Some notable examples include the Loma Prieta $M_{S} 7.1$ earthquake on $17 \mathrm{Oc}$ tober 1989 ( $f=0.01-10 \mathrm{~Hz}, D=7 \mathrm{~km}, A=1.5 \mathrm{nT}$; FraserSmith et al., 1990; Bernardi et al., 1991), as well as the Spitak $M_{S} 6.9$ earthquake on 7 December 1988 ( $f=0.005-1 \mathrm{~Hz}$, $D=200 \mathrm{~km}, A=0.2 \mathrm{nT}$; Molchanov et al., 1992; Kopytenko et al., 1993). In addition, the geo-electric potential enhance- 
ment appeared 1-19 days before five of all six earthquakes (EQs) with a magnitude $>5$, which occurred within $75 \mathrm{~km}$ in Japan and its duration and intensity were several minutes to $1 \mathrm{~h}$ with an amplitude of $0.01-0.02 \mathrm{mV} \mathrm{m}^{-1}$ (Uyeda et al., 2000). Qian et al. (2002) reported the observation of ULF signals generated from the Jiji earthquake on 21 September 1999 in Taiwan and recorded at many stations at distances of $300-900 \mathrm{~km}$ in southeast China. Similarly, Ohta et al. (2002) have reported the observation of ULF/extremely low frequency (ELF) emissions generated from the Taiwan earthquake on 21 September 1999 and recorded at Nakatsugawa station in Japan at a distance of up to $2000 \mathrm{~km}$.

A more notable example reported by $\mathrm{Li}$ et al. (2013) is the Wenchuan $M_{S} 8.0$ earthquake on 12 May 2008, a typical mid-crust, which resulted in great devastation and 69000 deaths. This earthquake was preceded by more than 1 month of increasing anomalous ULF emissions with a climax starting on 9 May, 3 days before the Wenchuan main shock $\left(f=0.1-10 \mathrm{~Hz}, D=1440 \mathrm{~km}, A=1.3 \mathrm{mV} \mathrm{m}^{-1}\right)$. More details can be seen in Sect. 4.

Many simulating rock-pressure experiments were carried out in order to understand the producing mechanism of the electromagnetic information associated with seismic activities. Laboratory experiments by Qian et al. $(1996,2003)$ and Hao et al. (2003) presented that electromagnetic signals are always recorded when rock samples are subjected to dynamic stresses. Shorter-period electromagnetic pulses appearing at the last stage of the experiment may be induced by instantaneous electric current of accumulated charges during the stress accelerating. The work of Freund (Freund and Wengeler, 1982; Freund, 2002, 2009, 2010; Freund and Sornette, 2007; Scoville et al., 2015) has gained a new insight into the production of current and electromagnetic signals in stressed rocks. As rocks come under stress, stresses cause slight displacements of mineral grains in the rocks, which in turn leads to the activation of peroxy defects that preferentially sit on or across grain boundaries. The peroxy breakup leads to positive holes, and the positive holes are able to flow from stressed to unstressed rock, traveling fast and far by way of a phonon-assisted electron hopping mechanism using energy levels at the upper edge of the valence band. A gabbro sample $30 \mathrm{~cm} \times 15 \mathrm{~cm} \times 10 \mathrm{~cm}$ from Shanxi, China, was used in this test and a $55 \mathrm{nA}$ current has recorded about $2 \mathrm{~s}$ before failure, with the load being at about $30000 \mathrm{lbs}(\sim 13600 \mathrm{~kg})$ and the maximum spike reaches $450 \mathrm{nA}$ when the main failure took place (Freund, 2009).

Up to now, no clear explanation has been given although several physical mechanisms have been proposed to interpret the generation of EM emissions and electrical currents observed either during seismic activity or in the laboratory experiments. These include the electrokinetic and magnetohydrodynamic, piezomagnetism, stress-induced variations in crustal conductivity, microfracturing, etc. (Draganov et al., 1991; Park, 1996; Fenoglio et al., 1995; Egbert, 2002; Simpson and Taflove, 2005). Whatever the physical mechanism of electromagnetic generation is, it is well established that, during rock experiments conducted under laboratory conditions, a strong electrical current is produced when rocks are stressed, especially at the stage of the main rupture.

As the development of satellite Earth observation (EO), there is an increasing amount of evidence that during some last stages of the long-term process of preparation, there could be a transfer of energy between lithosphere, atmosphere, and ionosphere, so as to introduce the concept of a lithosphere-atmosphere-ionosphere coupling (LAIC) among the three involved layers of the Earth (Pulinets et al., 1994, 2000; Hayakawa and Molchanov, 2002; Molchanov et al., 2004; Kamogawa, 2006). When we investigate electromagnetic emissions induced by an electrical current or a magnetic moment on the surface or beneath the Earth, the effect of the medium air, crustal as well as ionosphere should be taken into account since these three media consist of different conductivities and we need to consider a lithosphereatmosphere-ionosphere electromagnetic coupling (Cummer, 2000). Several tentative LAIC models have been constructed based on ground-based and ionospheric observations prior to strong earthquakes and the investigation of the influence of external electrical field on ionospheric parameters has been developed quickly (Pulinets and Ouzounov, 2011; Pulinets and Davidenko, 2014; Sorokin and Hayakawa, 2013, 2014; Kuo et al., 2011, 2014; Namgaladze et al., 2012; Zolotov et al., 2012; Zolotov, 2015). At the same time, the ionosphere plays an important role in electromagnetic propagation at ELF and very low frequency (VLF). The ground and the ionosphere are good electrical conductors and form a spherical Earth-ionosphere waveguide (Cummer, 2000). In addition, in the controlled-source electromagnetic (CSEM) method, widely used in petroleum exploration or mining, the ionospheric influence on EM fields should be considered when the distance between a large-scale and large-power fixed source and the receiver is up to $1000 \mathrm{~km}$. EM fields can be amplified in the ionosphere as it is shown when we use the analytical solution of Maxwell equations, as well as numerical ones of the "Earth-ionosphere" mode with a source on the Earth's surface or in the lower atmosphere (Fu et al., 2012; Li et al., 2010a, b, 2011; Xu et al., 2012).

Therefore, in comparison with an electromagnetic attenuation without an ionospheric effect, the point is to evaluate the ionospheric influence on the electromagnetic propagation when the distance between the epicenter and the observing location is up to $1000 \mathrm{~km}$ or more. Furthermore, the comparison between the observation distance reported by $\mathrm{Li}$ et al. (2013) $(D=1440 \mathrm{~km})$ and the length of the Wenchuan earthquake main rupture $L=\sim 150 \mathrm{~km}$ (Zhang et al., 2009a) indicates that the length of the dipole source is not negligible. So in this paper, based on the work of Key (2009), a three-layer (Earth-air-ionosphere) physical model as well as a two-layer (Earth-air) model, containing a finite length dipole current source co-located along the fault and beneath the Earth, is introduced in Sect. 2. For specified parameters, 


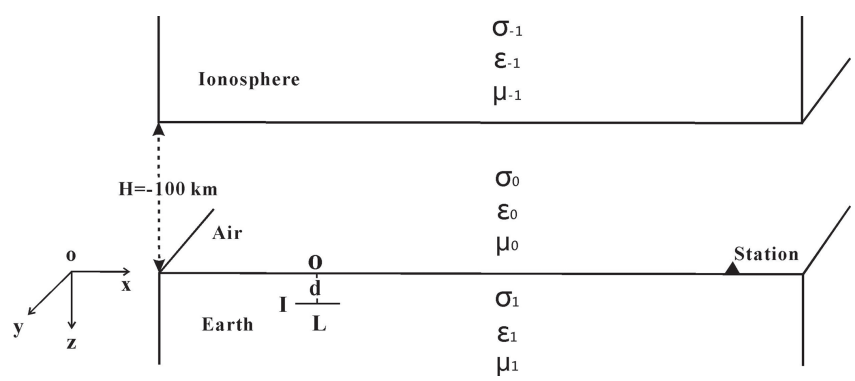

Figure 1. An $x$-directed dipole current source, with its central coordinate $(0,0, d)$, is placed in the bottom medium (Earth) of a three layer modeling (Earth-air-ionosphere model), where $z$ is defined positive in the downward direction.

some simulation results of the current source with and without ionospheric effects are given in Sect. 3. In Sect. 4, using assumed parameters, the simulation results for the case of the Wenchuan earthquake reported by Li et al. (2013) are presented. Discussion and conclusions are given in Sects. 5 and 6 , respectively.

\section{Description of the modeling methodology}

In order to study the electromagnetic fields emitted by a long dipole current source, the approach used here follows the magnetic vector potential formulation described in Wait (1982) and developed by Key (2009), who generalized the formulation to allow for multiple layers above the transmitter (in addition to multiple layers below). He used exponential forms for the recursions rather than hyperbolic functions in isotropic media, which consists of $N$ layers of isotropic conductivity $\sigma_{i}$ where $i=1, \ldots, N$, and which uses a right-handed coordinate system with the $z$ axis pointing down. Assuming a time-harmonic source with $e^{-i \omega t}$ time dependence, negligible magnetic permeability $\mu$ variations, and angular frequencies $\omega$ that are low enough so that displacement currents can be neglected, Maxwell's equations are

$\nabla \times E=i \omega B$

and

$\nabla \times B=\mu \sigma E+\mu J_{s}$.

Expression $J_{s}=\boldsymbol{I} \delta\left(r-r_{0}\right)$ is the imposed electric dipole source at position $r_{0}$ with vector moment $\boldsymbol{I}$, and here is restricted to an infinitesimal dipole with unit moment.

Based on the model setup by Key (2009), some modifications will be done in this study in order to complete the target here. A physical model is specified. It has three layers, Earth, air, and ionosphere, which is called Earth-air-ionosphere model. Its coordinate system is denoted in Fig. 1 with $z$ direction being downward. An $x$-directed dipole of a length $L$ and a current $I$ is placed in the bottom medium (Earth: $z>0$ ), which is homogeneous and has the electrical properties: magnetic permeability $\mu_{1}$, permittivity $\varepsilon_{1}$, and conductivity $\sigma_{1}$. The middle medium (air: $-100 \mathrm{~km}<z<0$ ) is described by its electrical properties $\mu_{0}$, $\varepsilon_{0}$ (i.e., $8.854 \times 10^{-12}$ Farad $^{-1}$ ) and $\sigma_{0}$ (i.e., $10^{-14} \mathrm{~S} \mathrm{~m}^{-1}$ ). The top medium (ionosphere: $z<$ $-100 \mathrm{~km})$ is characterized by electrical properties $\mu_{-1}, \varepsilon_{-1}$ and $\sigma_{-1}$ (i.e., $10^{-5} \mathrm{~S} \mathrm{~m}^{-1}$ ).

As a comparison, a two-layer model (Earth-air model) including an Earth medium $(z>0)$, as well as an air medium $(z<0)$, is also established during the study. All the corresponding parameters described are the same as those of Earth-air-ionosphere model.

We assume that the total space is non-magnetic and that the magnetic permeability $\mu$ variations are negligible in the different layers; i.e., $\mu_{1}=\mu_{0}=\mu_{-1}=4 \pi \times$ $10^{-7}$ Farad $^{-1}$. However, the ionosphere, as the electrically conducting section of the upper atmosphere, plays such an important role for the electromagnetic propagation that we set $\varepsilon_{-1}=5 \varepsilon_{0}$ when an ionospheric effect on electromagnetic transmission is taken into consideration. On the other hand, we have $\varepsilon_{1}=\varepsilon_{0}=8.854 \times 10^{-12}$ Farad $^{-1}$; i.e., $\varepsilon_{1}$ is not considered as zero during all calculations. Under these conditions, the formula listed above is still suitable and more explanations about the potential formulation of a horizontal electric dipole can be found in the Appendix A of Key (2009), and related programs are available with access to the website (http://marineemlab.ucsd.edu/). The horizontal finite length dipole source can be viewed as integral for an infinitesimal horizontal dipole during related calculations.

\section{Simulation results}

According to these two models presented above, several free parameters must be specified in order to investigate the attenuation characteristics of the electromagnetic fields emitted by a long $x$-directed dipole current source. As for the parameters of the dipole current source, we select $L=150 \mathrm{~km}$, the Wenchuan earthquake main rupture stage within $30 \mathrm{~s}$ out of $90 \mathrm{~s}(\sim 300 \mathrm{~km})$ based on Zhang et al. (2009a, Fig. 1), the depth $d=19 \mathrm{~km}$ (Xu, 2009), the hypocenter depth of the Wenchuan case and the current is set to be $I=1 \mathrm{~A}$ temporarily. Here, the Earth is considered to be an isotropic media with an average conductivity $\sigma_{1}$, and we assume $\sigma_{1}=$ $1.0 \times 10^{-3} \mathrm{~S} \mathrm{~m}^{-1}$ at this time; i.e., $\rho_{1}=10^{3} \mathrm{ohm} \mathrm{m}$. Furthermore, the ground conductivity depends not only on the local petrology but also on the porosity, temperature, and pressure (e.g., Wait, 1966). All these parameters are common to two models. The parameter $\varepsilon_{-1}=5 \varepsilon_{0}$ is very important during the calculation in the three-layer model whereby it can potentially affect the transmission of electromagnetic waves produced by the dipole beneath the Earth, and possibly induce the Earth-atmosphere-ionosphere electromagnetic coupling.

Figure $2 \mathrm{a}-\mathrm{d}$ display electric field amplitude $\left|E_{x}\right|$ decay curves along the $x$ axial direction with the frequen- 

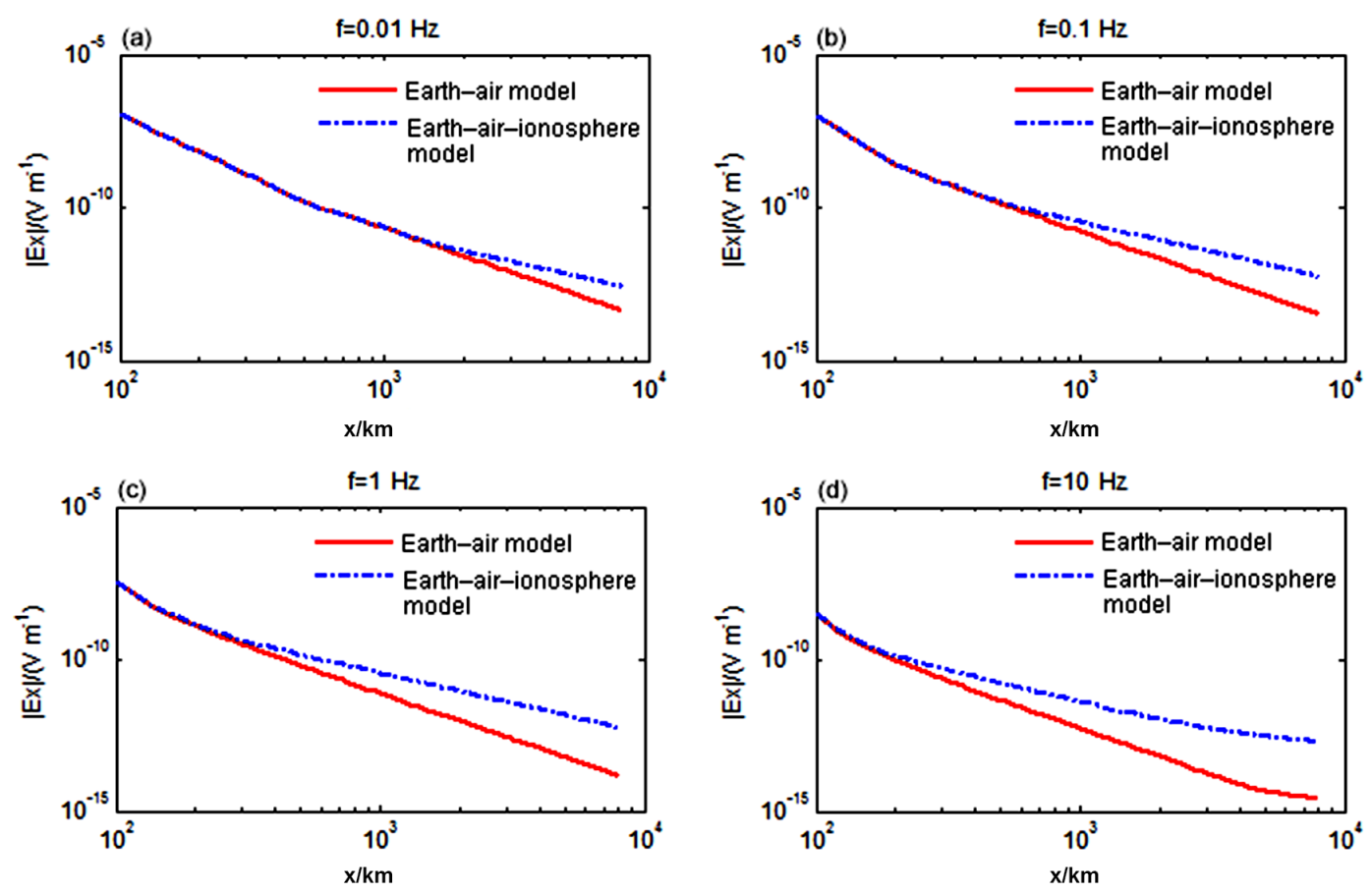

Figure 2. Electric field $\left|E_{x}\right|$ decay curves along $x$ axial direction as a function of the observing distance for the Cartesian coordinate system with different frequencies. Red solid lines stand for electric field curves for the Earth-air model and blue dotted lines denote electric field curves with the ionospheric effect for the Earth-air-ionosphere model. (a) Total $\left|E_{x}\right|$ for $f=0.01 \mathrm{~Hz}$, (b) total $\left|E_{x}\right|$ for $f=0.1 \mathrm{~Hz}$, (c) total $\left|E_{x}\right|$ for $f=1 \mathrm{~Hz}$, and (d) total $\left|E_{x}\right|$ for $f=10 \mathrm{~Hz}$.

cies $f=0.01 \mathrm{~Hz}, f=0.1 \mathrm{~Hz}, f=1 \mathrm{~Hz}$, and $f=10 \mathrm{~Hz}$ for the Cartesian coordinate system up to $\sim 10000 \mathrm{~km}$ on the Earth's surface.

It can be seen from Fig. 2a-d that, first, the electrical field with high frequency has a bigger attenuation although all curves for both the Earth-air model (red solid lines) and the Earth-air-ionosphere model (blue dotted lines) decay rapidly as the distance increases. Second, each group of curves run at the same level for one fixed frequency, e.g., $f=1 \mathrm{~Hz}$, when an observing point is located at a relatively close distance, $\sim 300 \mathrm{~km}$ for $f=1 \mathrm{~Hz}$ (Fig. 2c) for example. That is to say, the ionospheric influence on electromagnetic field transmissions can be neglected within this range. However, this range changes for different frequencies and it becomes smaller as the operating frequency of the current source increases (e.g., more than $1000 \mathrm{~km}$ for $f=0.01 \mathrm{~Hz}$; Fig. 2a and only $\sim 200 \mathrm{~km}$ for $f=10 \mathrm{~Hz}$; Fig. $2 \mathrm{~d}$ ). Third, the most important result is, as the distance increases, field curves with an ionospheric effect (blue dotted lines) run along a different path from that of curves without an ionospheric effect (red solid lines) and the ionospheric lines attenuate more slowly. Now, this kind of ionospheric influence can no longer be neglected. The ionospheric difference is about 1 magnitude $(\times 10)$ for all the frequencies listed and even once up to 2 magnitudes for $f=10 \mathrm{~Hz}$ within the range shown in Fig. 2. For example, the ionospheric difference value shows
1 magnitude from $\sim 840 \mathrm{~km}$ and up to 2 magnitudes from $\sim 3700 \mathrm{~km}$ for $f=10 \mathrm{~Hz}$ (Fig. 2d).

\section{The Wenchuan $M_{S} 8.0$ earthquake as a sample}

\subsection{Estimating the seismo-telluric current magnitude}

The Hebei ULF $(0.1-10 \mathrm{~Hz})$ electromagnetic observation network was constructed at the beginning of 1980s after the occurrence of the 28 July 1976, Tangshan $M_{S} 7.8 \mathrm{EQ}$, with the aim of monitoring fluctuations in electromagnetic radiations before seismic activities mainly around Beijing. More details of the observation system can be found in Zhuang et al. (2005) and Li et al. (2013). The system measures electrical signals and a DJ-1 recorder is employed to record the potential difference between two electrodes (SN: south-north and EW: east-west). The recording method uses an analog automatic real-time continuous pen recorder with a speed of $1 \mathrm{~mm} \mathrm{~s}^{-1}$. In general, there are only parallel lines with perpendicular automatic clock marked signals on the record paper around a drum and six lines are left per hour. A blank recording paper replaces the recorded one at 09:00 local time (LT) everyday (seen Fig. 3).

During the period from January 2007 to December 2008, electrical emissions were recorded at three among four stations (only four stations, Gaobeidian, Ningjin, Qingxian and 


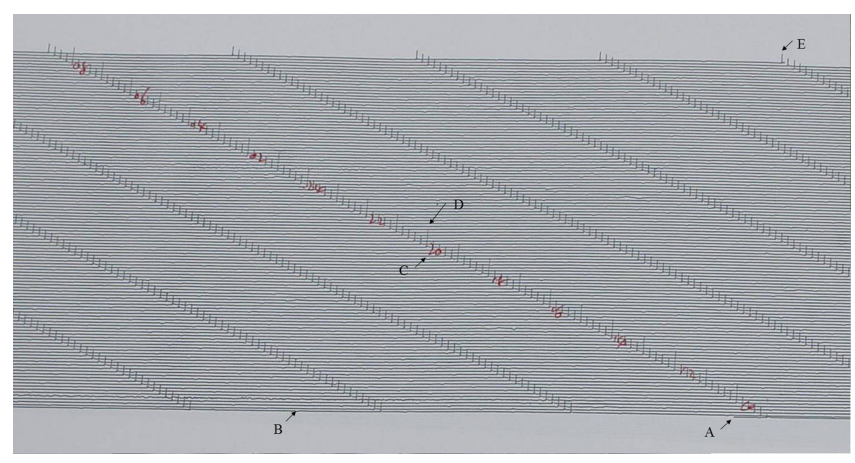

Figure 3. Copy of a part of the normal original record (EW component) from 09:00 LT 1 May to 09:00 LT 2 May 2008 at Gaobeidian station. Corresponding letters: A denotes a start record point, i.e., 09:00 LT 1 May; B is a normal record line; $\mathrm{C}$ indicates a manually marked time, i.e., 20:00 LT 1 May; D indicates an automatic marked perpendicular hour line, i.e., 20:00 LT 1 May; and E shows an end record point, i.e., 09:00 LT 2 May.

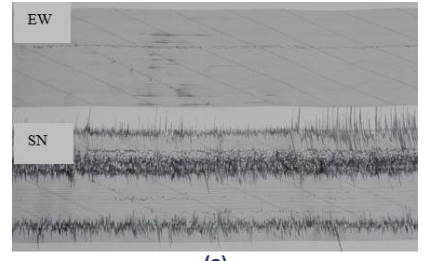

(a)

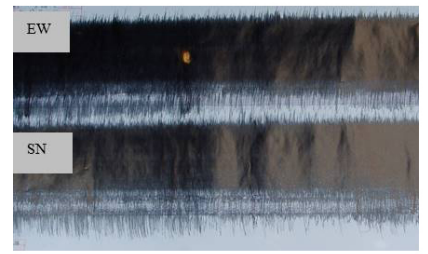

(c)

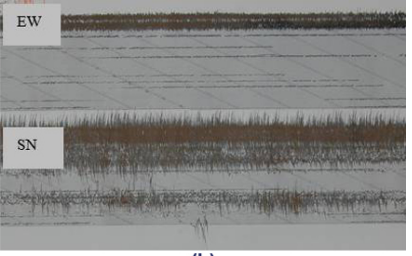

(b)

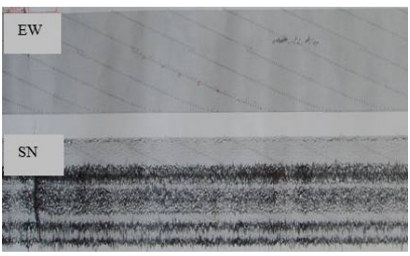

(d)
Figure 4. Part of real-time recordings at Gaobeidian station (a) from 09:00 LT 13 February to 09:00 LT 14 February 2008, (b) from 09:00 LT 8 May to 09:00 LT 9 May 2008, (c) from 09:00 LT 12 May to 09:00 LT 13 May 2008, and (d) from 09:00 LT 20 May to 09:00 LT 21 May 2008.

Sanhe, ran normally during this time period; Fig. 5) and the recording at Gaobeidian station shows typical fluctuation characteristic. Anomalous emissions first appeared at the end of October 2007 and the information was not recorded everyday while it mainly accumulated in $\mathrm{SN}$ direction like what is shown in Fig. 4a. This kind of situation lasted till the beginning of April 2008, from when relative high frequency and large amplitude signals were recorded almost every day with a persistent time. On 9 May 2008, 3 days before the Wenchuan $M_{S}$ 8.0 EQ, the amplitudes of signals were suddenly subjected to an abrupt enhancement at the same time, during 05:00-07:00 LT, both in SN and in EW directions (Fig. 4b) and the abnormity reached the climax stage $\left(\sim 1.3 \mathrm{mV} \mathrm{m}^{-1}\right.$ for electric field, like Fig. $\left.4 \mathrm{c}\right)$ lasting till 17 May 2008. After 18 May the total signal amount decreased sharply and the characteristics of the signals at this stage are shown in Fig. 4d, more like that before April 2008. The SN information lasted till the end of September 2008 except for high emissions appearing before several powerful aftershocks. It is the first time that the abnormality is with such a large amplitude and such a long duration in the observation history of this network although several strong EQs were recorded before (Li et al., 2013).

On the base of the work of rock experiments conducted under laboratory conditions, there is a reason to believe that a giant seismo-telluric current was generated when the main rupture took place during the Wenchuan earthquake on 12 May 2008 and that this current mainly propagated along the Longmenshan fault. At the same time a strong electrical field induced by this current suddenly increased. This electrical field was recorded at the ground-based Gaobeidian ULF observing station, $1440 \mathrm{~km}$ away from the epicenter of the shock, with a SN maximum amplitude of $70 \mathrm{~mm}$, i.e., $1.3 \mathrm{mV} \mathrm{m}^{-1}$ (Li et al., 2013); that is, $E_{\mathrm{obs}(\mathrm{SN})}=1.3 \mathrm{mV} \mathrm{m}^{-1}$ in the following statement (Fig. 5).

In order to establish a relationship between the seismotelluric current during the main event and the observable ground electrical signals registered at Gaobeidian station, we consider that a finite length current dipole source, with the length being the main rupture $L=150 \mathrm{~km}$ of the Wenchuan earthquake and the current $I$, is co-located with the Longmenshan main fault ( $x$ direction), with the depth being $d=19 \mathrm{~km}$. Then one can refer to Fig. 1 with an ionospheric effect.

Corresponding to Fig. 1, a coordinate system on the Earth's surface (see Fig. 5) is set up to calculate the observable electrical field along the $x$ direction $E_{\text {obs }}$ according to the electrical value $E_{\mathrm{obs}(\mathrm{SN})}=1.3 \mathrm{mV} \mathrm{m}^{-1}$ recorded at the Gaobeidian station. The Gaobeidian station lies in the extension line of the Longmenshan fault, which trends northeast and dips about $60^{\circ} \mathrm{W}(\mathrm{Xu}, 2009)$. Other locations of stations are shown in Fig. 1 of Li et al. (2013) and here they are shown in Fig. 5, which includes a ground surface coordinate system. From Fig. 5, we see that the electrical filed component intensity along the $x$ direction is about $\left|E_{x}\right|=E_{\mathrm{obs}}=1.5 \mathrm{mV} \mathrm{m}^{-1}$ $\left(E_{\mathrm{obs}(\mathrm{SN})}=\sin 60^{\circ} \times E_{\mathrm{obs}}=1.3 \mathrm{mV} \mathrm{m}^{-1} \rightarrow\right.$ $E_{\text {obs }}=1.5 \mathrm{mV} \mathrm{m}^{-1}$ ).

As the observing frequency of the electromagnetic observation system is $0.1-10 \mathrm{~Hz}$ and the recorder belongs to a real-time analog record, it is not easy to figure out the right frequency of the signals registered at the Gaobeidian station during the maximum stage prior to the Wenchuan earthquake. We set the main frequency $f=1 \mathrm{~Hz}$ during our calculations although the information is of a short period $\sim 0.1-$ $0.3 \mathrm{~s}$ and a large amplitude $\sim 1.3 \mathrm{mV} \mathrm{m}^{-1}$ ( $\mathrm{Li}$ et al., 2013), and frequency bands (0.4-3 and 0.05-0.1 s) with various amplitudes were observed (Guan et al., 2003). At the same time, the results of 2-D MT inversion in the Longmenshan fault 


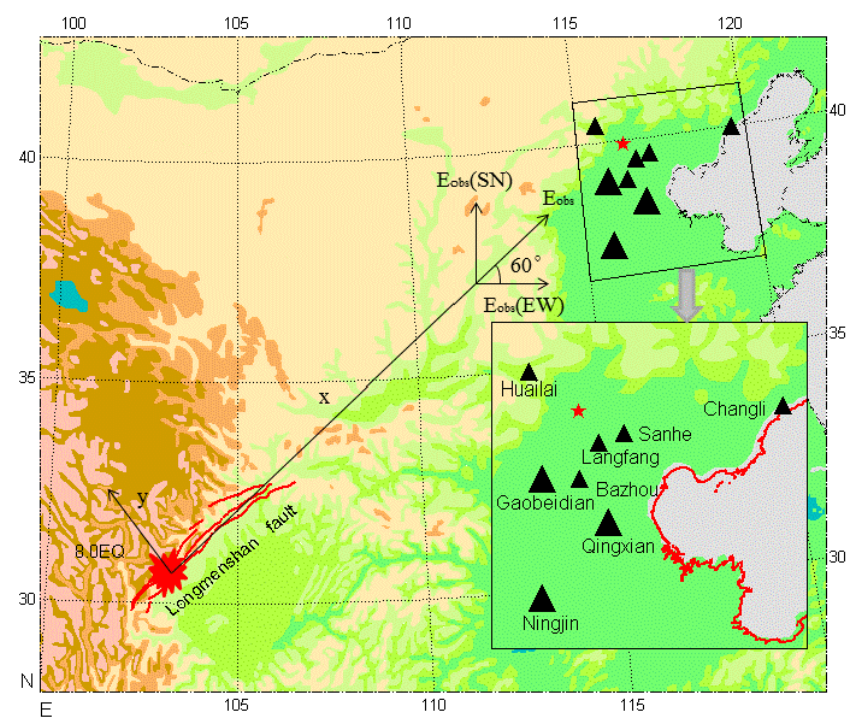

Figure 5. Distribution of the Wenchuan earthquake epicenter and observation stations. Black solid triangles present the related locations of observation stations in Hebei electromagnetic observation network, bigger ones indicate the stations where abnormal information was recorded and the red star denotes Beijing (Li et al., 2013, Fig. 1). A ground surface coordinate system is added.

show that the apparent resistivity logarithm is $\sim 1-4.8$ (Zhu et al., 2008) and it is a wide range.

Figure 6 shows the calculated values of $\left|E_{x}\right|$, expected at the observation location $(1440 \mathrm{~km}, 0,0)$ due to a dipole source of $L=150 \mathrm{~km}, I=1 A$, and $d=19 \mathrm{~km}$ (Fig. 1), as a function of the typical crustal material's conductivity $\sigma$. Comparing the red line with the blue dotted line, the ionospheric effect is clearly displayed throughout the variation of the crustal conductivity. A rapid attenuation (in excess of 20 of magnitude) of the field values indicates the importance of the conductivity $\sigma$. It is difficult to specify the average conductivity $\sigma$ (referred to as $\sigma_{1}$ in the context) of the homogeneous Earth medium, even for the typical Wenchuan area. However, combined with $f=1 \mathrm{~Hz}$ here, the skin depth depends on the conductivity $\sigma$, given by the formula $\delta=\left(\pi f \mu_{0} \sigma\right)^{-1 / 2}$. Taking the depth $d=19 \mathrm{~km}$ into account, here $\delta=d=19 \mathrm{~km}$ and the calculated $\sigma_{1}$ is attained, i.e., $\sigma_{1}=7.0 \times 10^{-4} \mathrm{~S} \mathrm{~m}^{-1}$, which is advantageous to radiate electromagnetic waves within this depth.

Using the same parameters as above, the simulation results show that the seismo-telluric current along the main fault needed to produce an electrical ground signal $E_{\mathrm{obs}(\mathrm{SN})}=1.3 \mathrm{mV} \mathrm{m}^{-1}$ at the Gaobeidian station when the Wenchuan event occurred, is about $5.0 \times 10^{7} A$ with the ionospheric effect and $3.7 \times 10^{8} \mathrm{~A}$ without the ionospheric effect. As it is expected, these two results have 1 magnitude $(\times 10)$ difference from each other. While the former is more reasonable under these conditions because the seismo-telluric current produced by the Wenchuan main rupture is specified.

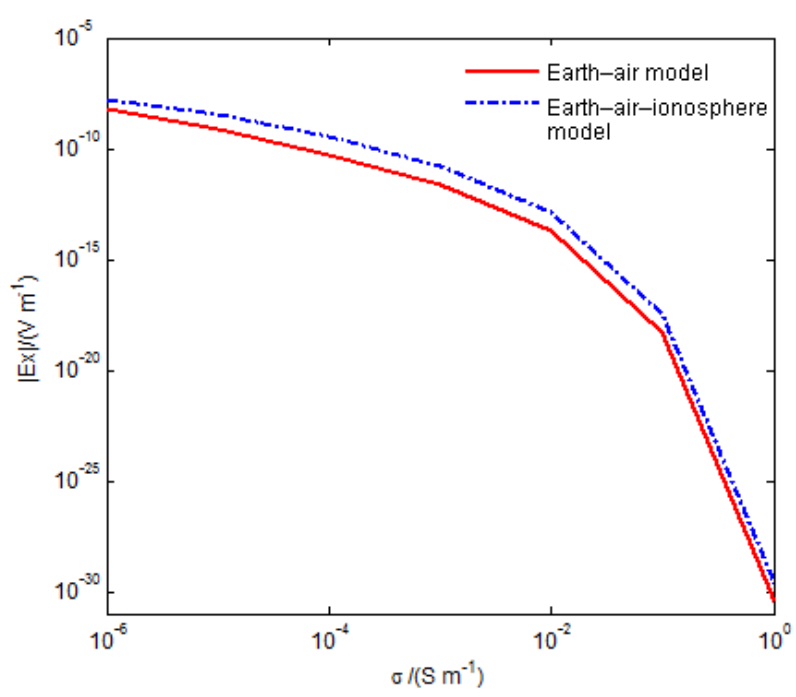

Figure 6. The calculated value of $\left|E_{x}\right|$, expected at the observation location $(1440 \mathrm{~km}, 0,0)$ due to a dipole source of $L=150 \mathrm{~km}, I=$ $1 A, f=1 \mathrm{~Hz}$, and $d=19 \mathrm{~km}$ (Fig. 1), as a function of the typical crustal material's conductivity $\sigma$ both in the Earth-air model (red line) and the Earth-air-ionosphere model (blue dotted line).

\subsection{Detectability under the ionospheric effect}

Now according to the Wenchuan earthquake example, the seismo-telluric current source $(f=1 \mathrm{~Hz}, d=19 \mathrm{~km}$, $L=150 \mathrm{~km}$, and a current $I=5.0 \times 10^{7} \mathrm{~A}$ considering the Earth-air-ionosphere model) is thought of as a powerful finite length dipole source.

Figure 7 displays the fluctuations of the surface electrical fields with and without ionospheric effects for the Wenchuan source along $x$ axial direction. It shows no obvious ionospheric effect within $300 \mathrm{~km}$, while this effect is roughly up to 1 order of magnitude from $\sim 800 \mathrm{~km}$. The gap becomes larger as the distance increases, near 2 magnitudes from $\sim 9000 \mathrm{~km}$, and then it keeps this gap till $10000 \mathrm{~km}$. Under this condition, if the resolution of an observing system is $0.1 \mathrm{mV} \mathrm{m}^{-1}$, the recorded distance of such a signal must be $\sim 5400 \mathrm{~km}$ (blue arrow) with an ionospheric effect, or it is only $\sim 1800 \mathrm{~km}$ (red arrow) without an ionospheric effect. So the ionosphere facilitates the electromagnetic wave propagation, as if the detectability of the system were improved effectively, and it would be easier to record a signal even at stations located beyond their detectability thresholds.

\subsection{Wave 2-D distribution}

We perform electromagnetic wave fields for the Wenchuan source and this is done in the ground plane region $-1000 \mathrm{~km}<x<1000 \mathrm{~km}$ and $-1000 \mathrm{~km}<y<1000 \mathrm{~km}$ in order to visualize the 2-D distribution of the wave power surrounding the electrical source. 


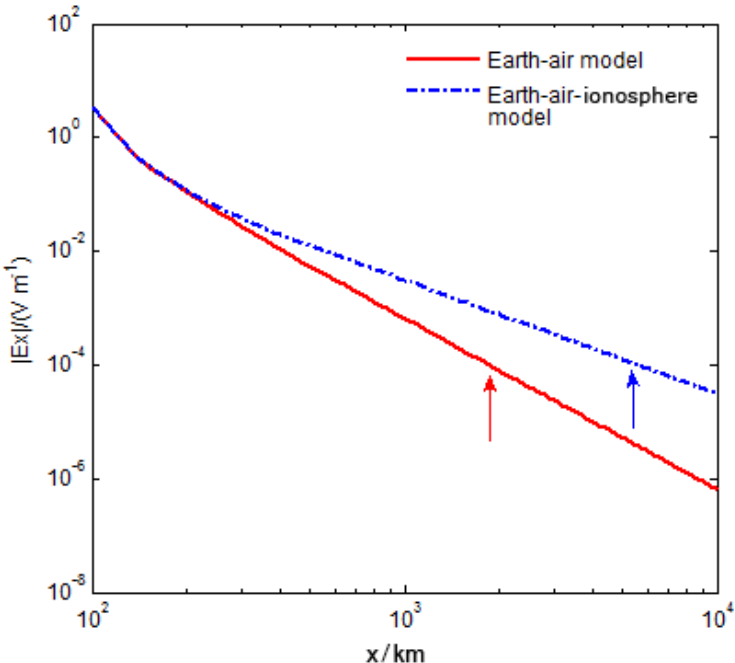

Figure 7. The Wenchuan source producing electric field $\left|E_{x}\right|$ decay curves as a function of the distance along $x$ axial direction with an ionospheric effect (blue dotted line), as well as without an ionospheric effect (red line). The electric field $\left|E_{x}\right|=0.1 \mathrm{mV} \mathrm{m}^{-1}$ is labeled with a red and a blue arrow.

Figure 8 displays the 2-D power distributions of the electrical field components $\left|E_{x}\right|,\left|E_{y}\right|$, and the total $|E|$ $\left(|E|^{2}=\left|E_{x}\right|^{2}+\left|E_{y}\right|^{2}\right)$ after making a logarithm calculation on the Earth's surface. It can be seen firstly from Fig. 8a that there is an obvious constant strong power along the current element length $(-75 \mathrm{~km}<x<75 \mathrm{~km})$ in the $x$ direction. The electrical value in this area is not discussed here because it is usually considered not precise. Then the strong field radiates outward surrounding four main axes, indicating firstorder rough decay of the field at $\sim 160 \mathrm{~km}, 2$ orders of magnitude at $\sim 320 \mathrm{~km}$ from the source endpoint in the $x$ direction. There is only 3 orders decay till $1000 \mathrm{~km}$ away because of the ionospheric facilitating effect on the field and it keeps a strong value $\left(\sim 1.86 \mathrm{mV} \mathrm{m}^{-1}\right)$, which can be fairly recorded by the stations. However, there are also weak power areas along lines, which form $45^{\circ}$ angle with the principal axis for the electrical field power $\left|E_{x}\right|$ (Fig. 8a). Complementally, the electrical field power $\left|E_{y}\right|$ (Fig. 8b) is basically characterized by strong power areas between two main axes, as well as weak ones along four chief axes. The power distribution of the total $|E|$ consequently appears to be symmetric to the center circle outside of the source (Fig. 8c), which also indicates that the radiating patterns of the electrical field power $\left|E_{x}\right|$ and the electrical field power $\left|E_{y}\right|$ are complementary (one is the strong area and the other is the weak area) to each other surrounding the source.

\section{Discussion}

In recent years, there has been an increasing amount of evidence that during the last stages of the long-term process of preparation, there could be a transfer of energy between the lithosphere and the above layers of the atmosphere and ionosphere, so as to introduce the concept of a LAIC among the three involved layers of the Earth system (Pulinets et al., 2000; Hayakawa and Molchanov, 2002; Molchanov et al., 2004; Pulinets and Ouzounov, 2011). On the one hand, the "energy source" is usually thought to be beneath the Earth's surface and related to tectonic activities in the lithosphere. On the other hand, numerous rock-pressure experiments and electromagnetic observations associated with seismic activities have already proved that a giant electrical current and an abrupt increase of electromagnetic signals occurs during the main rupture of stressed rocks. These phenomena happened on 9 May 2008, 3 days before the Wenchuan event, whose hypocenter is located in the mid-crust. The strong seismotelluric current is thought to run mainly along the Longmenshan fault and electromagnetic oscillations, induced by the current and predominated by ULF frequency band, propagate up to the ionosphere and give rise to perturbations of ionospheric parameters. Some of these parameters have been investigated, such as GPS TEC and f0F2 (Yu et al., 2009; Xu et al., 2010; Akhoondzadeh et al., 2010), DEMETER satellite O+ density (Zhang et al., 2009b), electron density and electron temperature (Zeng et al., 2009), etc. Fortunately, all of these study results present a climax on 9 May 2008 and this indicates a LAIC or interaction aroused by these electromagnetic signals prior to the Wenchuan event.

Unfortunately, at present, most of the investigations put emphasis on the effect of earthquakes upon the ionosphere and few of them pay attention to an inverse problem, that is, the ionospheric influence on the electromagnetic waves passing through.

The ionosphere, as a part of the electrical conducting region of the upper atmosphere, can enhance electromagnetic fields and cause the decay as a function of distance to slow down when an observation is beyond ionospheric range, and the ionospheric effect can be up to 1-2 orders of magnitude of the electrical fields in our simply three-layer model for some specified parameters we have selected here.

Considering the Wenchuan event, the electrical signals from the lithosphere interact with the ionosphere and are at the same time enhanced, and then registered at $1440 \mathrm{~km}$ Gaobeidian station with the amplitude of $\sim 1.3 \mathrm{mV} \mathrm{m}^{-1}$. This electrical field is used to simulate the seismo-telluric current produced by the Wenchuan main rupture in an Earthair-ionosphere model together with an Earth-air model. The results present that, the seismo-telluric currents with and without ionospheric effects must be about $5.0 \times 10^{7} \mathrm{~A}$ and $3.7 \times 10^{8} A$, respectively. Compared with the expected seismo-telluric current $\sim 10-100 \mathrm{kA}$ of the "Alum Rock" $M_{W}=5.6$ earthquake for an observed $30 \mathrm{nT}$ pulse at $1 \mathrm{~Hz}$ and $D=2 \mathrm{~km}$ (Bortnik et al., 2010), this result is probably in a reasonable range.

However, first, the total rupture of the Longmenshan fault during the Wenchuan main shock is extremely com- 


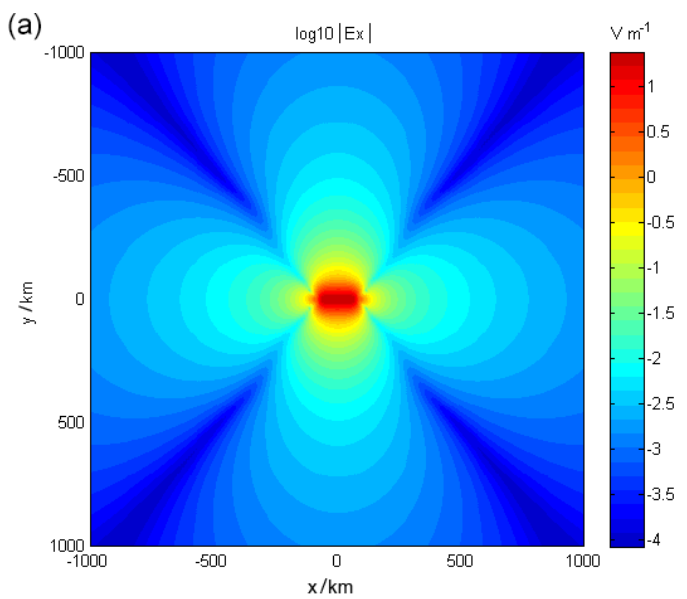

(b)
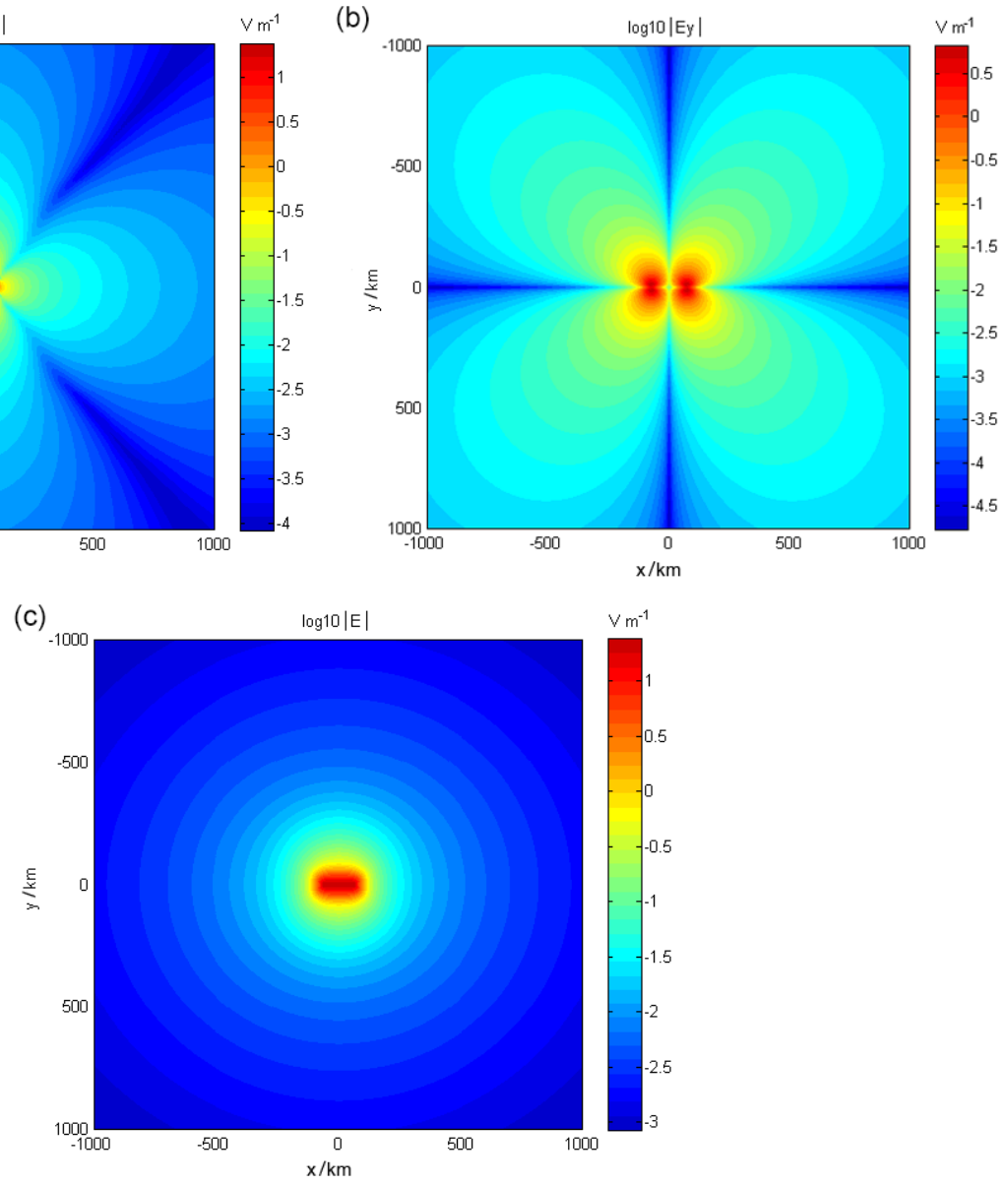

Figure 8. Two-dimensional distributions of electrical field power: (a) $\left|E_{x}\right|$, (b) $\left|E_{y}\right|$, and (c) total $|E|$ after a logarithm calculation for the Wenchuan source using an Earth-air-ionosphere model.

plicated, which is comprised of tenths of rupture stages and several pauses, totaling $90 \mathrm{~s}$ for the whole rupture process $(\sim 300 \mathrm{~km})$, according to Zhang et al. (2009a). Thus, the total surface rupture $\sim 300 \mathrm{~km}$ is nevertheless not used here. While performing the analysis on only the primary $30 \mathrm{~s}$, a main stage of the Wenchuan earthquake (out of $90 \mathrm{~s}$ as we have selected $L=150 \mathrm{~km}$ above) is expected to be representative of the majority of the rupture to generate a seismo-telluric current. Second, three media are thought of as a homogeneous isotropic medium in our models and with the same average conductivity value for each one, especially for the Wenchuan area. However, the Earth conductivity plays such an important role that it predominately affects the fluctuations of the electrical fields as shown in Fig. 6, although no one exactly knows the right conductivity of the Earth medium at the rupture depth. The value $\sigma_{1}=7.0 \times 10^{-4} \mathrm{~S} \mathrm{~m}^{-1}$, which has been used in all analyses, is estimated when the observing frequency range $f=0.1-$ $10 \mathrm{~Hz}$ and the hypocenter depth $d=19 \mathrm{~km}$ of the Wenchuan main event are taken into account for the skin-depth formula. One must also mention that we use $f=1 \mathrm{~Hz}$ in our calcu- lations because we cannot identify the actual frequencies in the recorded analog signals. All these can probably underscore our simulation results.

While these disadvantageous selections are maybe not so important at the same time, the key point of this paper is of the ionospheric influence on electromagnetic wave propagation and our investigation attains advantageous results.

The "selectivity" or "orientation" of the electromagnetic information is a very important characteristic during seismic activities (Varotsos and Lazaridou, 1991). For a finite length dipole source of the Wenchuan earthquake, its 2-D distributions of electrical field components $\left|E_{x}\right|$ and $\left|E_{y}\right|$, which are orthogonal each other, on the Earth's surface show there are strong field power areas and weak field power areas around the source as illustrated by Bortnik et al. (2010). The radiating pattern of the total $|E|$ in this investigation is symmetric to the center circle outside of the source, which indicates a signal is always registered to anyone direction if a system is designed to measure the total field $|E|$ or both $\left|E_{x}\right|$ and $\left|E_{y}\right|$ components instead of only one. This result also basically supports the practices of selectivity or orientation, the 
observing reality before the Wenchuan earthquake described by Li et al. (2013), for example, "compared with the EW (east-west) orientation, the electromagnetic signal is more obvious in the SN (south-north) orientation". The selectivity effect is a complex phenomenon that may be attributed to a superposition of the following three factors: "source characteristics", "travel path" and "inhomogeneities close to the station" (Varotsos and Lazaridou, 1991; Varotsos et al., 2005). Analytical solutions of Maxwell equations (Varotsos et al., 2000), as well as numerical ones (Sarlis et al., 1999), convince that selectivity results from the fact that earthquakes occur through a slip on faults, which are appreciably more conductive than the surrounding medium.

\section{Conclusions}

In this paper, a three-layer (Earth-air-ionosphere) physical model, as well as a two-layer (Earth-air) model, is employed to investigate the ionospheric effect on the wave fields for a finite length dipole current source co-located with the main fault of an earthquake when an observing location distance is up to $1000 \mathrm{~km}$ or even more. For a dipole source with specified parameters of the length $L=150 \mathrm{~km}$, the current $I=1 \mathrm{~A}$, and the depth $d=19 \mathrm{~km}$, the results show that all fields are free of the ionospheric effect for different frequencies in relative short ranges, e.g., $\sim 600 \mathrm{~km}$ for $f=0.1 \mathrm{~Hz}$, which implies the ionospheric influence on electromagnetic field transmissions can be neglected within this range. However, the ionosphere can increase the field amplitude and slow the decay when an observation is out of this range and the ionospheric effect can be up to 1-2 orders of magnitude of the electrical fields.

This is applicable to the 12 May 2008 Wenchuan $M_{S} 8.0$ earthquake during which a strong electromagnetic signal with an amplitude of $\sim 1.3 \mathrm{mV} \mathrm{m}^{-1}$ is recorded by the Gaobeidian ULF ( $f=0.1-10 \mathrm{~Hz})$ observing station $1440 \mathrm{~km}$ from the epicenter. The main fault rupture producing a current is equivalent to a finite length dipole current source, with a nucleation depth of $19 \mathrm{~km}$ and a length of $150 \mathrm{~km}$. Considering the Earth-air-ionosphere model, the expected current for the most typical properties of Wenchuan area is of $5.0 \times 10^{7} \mathrm{~A}$, which is 1 magnitude smaller than the current value of $3.7 \times 10^{8} \mathrm{~A}$ obtained with the Earth-air model free of an ionospheric effect. On the contrary, a signal produced by a seismic activity can be advantageously recorded by a remote station under the ionospheric effect as if the detectability of the system were improved effectively.

The 2-D power distributions of the electrical field components $\left|E_{x}\right|,\left|E_{y}\right|$, and the total $|E|$ after making a logarithm calculation on the Earth's surface are characterized by different radiating patterns. There are strong power areas along four main axes as well as weak power areas between two main axes for the electrical field $\left|E_{x}\right|$, whereas the component $\left|E_{y}\right|$ displays a complementary radiating pattern with strong areas and weak areas. Therefore, fortunately, a signal is always registered to any one direction if a system is designed to measure the total field $|E|$ (or both $\left|E_{x}\right|$ and $\left|E_{y}\right|$ components) as the radiating pattern of which is symmetric to the center circle outside of the source.

\section{Data availability}

The data presented in this paper are available by request through the following e-mail: limeixuxl@ seis.ac.cn.

Acknowledgements. The authors are grateful to the National Natural Science Foundation of China and this work was sponsored by the project Simulation and Interpretation of the Spatial Electromagnetic Phenomena Coupling before the Wenchuan $M_{S} 8.0$ Earthquake under grant agreement no. 41204057.

Edited by: C. Krawczyk

Reviewed by: F. Freund and one anonymous referee

\section{References}

Akhoondzadeh, M., Parrot, M., and Saradjian, M. R.: Electron and ion density variations before strong earthquakes $(M>6.0)$ using DEMETER and GPS data, Nat. Hazards Earth Syst. Sci., 10, 718, doi:10.5194/nhess-10-7-2010, 2010.

Bernardi, A., Fraser-Smith, A. C., McGill, P. R., and Villard Jr., O. G.: Magnetic field measurements near the epicenter of the $M_{S} 7.1$ Loma Prieta earthquake, Phys. Earth Planet Interiors, 68, 45-63, 1991.

Bortnik, J., Bleier, T. E., Dunson, C., and Freund, F.: Estimating the seismotelluric current required for observable electromagnetic ground signals, Ann. Geophys., 28, 1615-1624, doi:10.5194/angeo-28-1615-2010, 2010.

Cummer, S. A.: Modeling Electromagnetic Propagation in the Earth-Ionosphere Waveguide, IEEE T. Antenn. Propag., 48, 212,2000

Draganov, A. B., Inan, U. S., and Taranenko, Y. N.: ULF magnetic signatures at the Earth due to groundwater flow: a possible precursor to earthquakes, Geophys. Res. Lett., 18, 1127-1130, 1991.

Eftaxias, K., Kapiris, P., Polygiannakis, J., Peratzakis, A., Kopanas, J., Antonopoulos, G., and Rigas, D.: Experience of short term earthquake precursors with VLF-VHF electromagnetic emissions, Nat. Hazards Earth Syst. Sci., 3, 217-228, doi:10.5194/nhess-3-217-2003, 2003.

Egbert, G. D.: On the generation of ULF magnetic variations by conductivity fluctuations in a fault zone, Pure Appl. Geophys., 159, 1205-1227, 2002.

Fenoglio, M. A., Johnston, M. J. S., and Byerlee J. D.: Magnetic and electric fields associated with changes in high pore pressure in fault zones: application to the Loma Prieta ULF emissions, J. Geophys. Res., 100, 951-958, 1995.

Fraser-Smith, A. C., Bernardi, A., McGill, P. R., Ladd, M. E., Helliwell, R. A., and Villard Jr., O. G.: Low-frequency magnetic measurements near the epicenter of the $M_{S} 7.1$ Loma Prieta earthquake, Geophys. Res. Lett., 17, 1465-1468, 1990. 
Freund, F.: Charge generation and propagation in igneous rocks, J. Geodynamics, 33, 543-570, 2002.

Freund, F.: Stress-activated positive hole charge carriers in rocks and the generation of pre-earthquake signals, in: Electromagnetic Phenomena Associated with Earthquakes, edited by: Hayakawa, M., Transworld Research Network, Trivandrum, India, 3, 41-96, 2009.

Freund, F.: Toward a unified solid state theory for pre-earthquake signals, Acta Geophys., 58, 719-766, 2010.

Freund, F. and Wengeler, H.: The infrared spectrum of $\mathrm{OH}^{-}$compensated defect sites in $\mathrm{C}-$ doped $\mathrm{MgO}$ and $\mathrm{CaO}$ single crystals, J. Phys. Chem. Solids, 43, 129-145, 1982.

Freund, F. and Sornette, D.: Electro-magnetic earthquake bursts and critical rupture of peroxy bond networks in rocks, Tectonophysics, 431, 33-47, 2007.

Fu, C. M., Di, Q. Y., Xu, C., and Wang, M. Y.: Electromagnetic fields for different type sources with effect of the ionosphere, Chinese J. Geophys., 55, 3958-3968, doi:10.6038/j.issn.00015733.2012.12.008, 2012 (in Chinese with English abstract).

Guan, H. P., Han, F. Y., Xiao, W. J., and Chen, Z. Y.: ULF electromagnetic observation and data processing methods, Earthquake, 23, 5-93, 2003 (in Chinese with English abstract).

Hayakawa, M. and Molchanov, O. A. (Eds.): SeismoElectromagnetics: Lithosphere-Atmosphere-Ionosphere Coupling, Tokyo, Japan: TERRAPUB, 2002.

Hao, J., Qian, S., Zhou, J., and Zhu, T.: ULF electric and magnetic anomalies accompanying the cracking of rock sample, Acta Seismol. Sin., 25, 102-111, 2003 (in Chinese with English abstract).

Kamogawa, M.: Pre-seismic lithosphere-atmosphere-ionosphere coupling, Eos, 87, 417-424, 2006.

Key, K.: 1-D inversion of multicomponent, multi-frequency marine CSEM data: Methodology and synthetic studies for resolving thin resistive layers, Geophysics, 74, F9-F20, 2009.

Kopytenko, Y. A., Matiashvili, T. G., Voronov, P. M., Kopytenko, E. A., and Molchanov, O. A.: Detection of ultra-low frequency emissions connected with the Spitak earthquake and its aftershock activity, based on geomagnetic pulsations data at Dusheti and Vardzia observatories, Phys. Earth Planet. Interiors, 77, 8595, 1993.

Kuo, C. L., Huba, J. D., Joyce, G., and Lee, L. C.: Ionosphere plasma bubbles and density variations induced by preearthquake rock currents and associated surface charges, J. Geophys. Res., 116, A10317, doi:10.1029/2011JA016628, 2011.

Kuo, C. L., Lee, L. C., and Huba, J. D.: An improved coupling model for the lithosphere-atmosphere-ionosphere system, J. Geophys. Res.-Space, 119, 3189-3205, 2014.

Li, M., Lu, J., Parrot, M., Tan, H., Chang, Y., Zhang, X., and Wang, Y.: Review of unprecedented ULF electromagnetic anomalous emissions possibly related to the Wenchuan $M_{S}=8.0$ earthquake, on 12 May 2008, Nat. Hazards Earth Syst. Sci., 13, 279286, doi:10.5194/nhess-13-279-2013, 2013.

Li, D., Di, Q. Y., and Wang, M. Y.: One-dimensional electromagnetic fields forward modeling for "Earth-ionosphere" mode, Chinese J. Geophys., 54, 2375-2388, doi:10.3969/j.issn.00015733.2011.09.021, 2011 (in Chinese with English abstract).

Li, Y., Lin, P. R., Zheng, C. J., Shi, F. S., Xu, B. L., and Guo, P.: The electromagnetic response modeling of the ELF method and the influence of the ionosphere, Geophysical \& Geochemical Exploration, 34, 332-339, 2010a (in Chinese with English abstract).
Li, D. Q., Di, Q. Y., and Wang, M. Y.: Study of large scale large power control source electromagnetic with "Earthionosphere" mode, Chinese J. Geophys., 53, 411-420, doi:10.3969/j.issn.0001-5733.2010.02.019, 2010b (in Chinese with English abstract).

Molchanov, O., Fedorov, E., Schekotov, A., Gordeev, E., Chebrov, V., Surkov, V., Rozhnoi, A., Andreevsky, S., Iudin, D., Yunga, S., Lutikov, A., Hayakawa, M., and Biagi, P. F.: Lithosphereatmosphere-ionosphere coupling as governing mechanism for preseismic short-term events in atmosphere and ionosphere, Nat. Hazards Earth Syst. Sci., 4, 757-767, doi:10.5194/nhess-4-7572004, 2004.

Molchanov, O. A., Kopytenko, Y. A., Voronov, P. M., Kopytenko, E. A., Matiashvili, T. G., Fraser-Smith, A. C., and Bernardi, A.: Results of ULF Magnetic field measurements near the epicenters of the Spitak $\left(M_{S} 6.9\right)$ and Loma Prieta $\left(M_{S} 7.1\right)$ earthquakes: comparative analysis, Geophys. Res. Lett., 19, 1495-1498, 1992.

Namgaladze, A. A., Zolotov, O. V., Karpov, M. I., and Romanovskaya, Y. V.: Manifestations of the earthquake preparations in the ionosphere total electron content variations, Nat. Sci., 4, 848-855, 2012.

Ohta, K., Umeda, K., Watanabe, M., and Hayakawa, M.: Relationship between ELF magnetic field and Taiwan earthquake, in: Lithosphere-Atmosphere-Ionosphere Coupling, edited by: Hayakawa, M. and Molchanov, O. A., Terra Science Publishers, Tokyo, 233-237, 2002.

Park, S. K.: Precursors to earthquakes: seismo-electromagnetic signals, Surv. Geophys., 17, 493-516, 1996.

Pulinets, S. A. and Ouzounov, D.: Lithosphere-AtmosphereIonosphere Coupling (LAIC) model-An unified concept for earthquake precursors validation, J. Southeast Asian Earth Sci., 41, 371-382, 2011.

Pulinets, S. A. and Davidenko, D.: Ionospheric precursors of earthquakes and Global Electric Circuit, Adv. Space Res., 53, 709723, 2014.

Pulinets, S. A., Legen'ka, A. D., and Alekseev, V. A.: Preearthquakes effects and their possible mechanisms. In: Dusty and Dirty Plasmas, Noise and Chaos in Space and in the Laboratory, Plenum Publishing, New York, 545-557, 1994.

Pulinets, S. A., Boyarchuk, K. A., Hegai, V. V., Kim, V. P., and Lomonosov, A. M.: Quasielectrostatical model of atmospherethermosphere-ionosphere coupling, Adv. Space Res., 26, 1209 1218, 2000.

Qian, S., Ren K., and Lü, Z.: Experimental study on VLF, MF, HF and VHF electromagnetic radiation characteristics with the rock breaking, Earthquake Science, 18, 346-351, 1996 (in Chinese with English abstract).

Qian, S., Hao, J., Zhou, J., and Gao, J.: Simulating experimental study on ULF electromagnetic precursors before Jiji $M_{S}=7.4$ earthquake, in: Lithosphere-Atmosphere-Ionosphere Coupling, edited by: Hayakawa, M. and Molchanov, O. A., Terra Science Publishers, Tokyo, 49-53, 2002.

Qian, S., Hao, J., Zhou, J., and Gao, J.: Precursory Electric and Magnetic Signals at ULF and LF Bands during the Fracture of Rocks under Pressure, Earthquake Research in China, 19, 109116, 2003 (in Chinese with English abstract).

Sarlis, N., Lazaridou, M., Kapiris, P., and Varotsos, P.: Numerical model of the selectivity effect and the V/L criterion, Geophys. Res. Lett., 26, 3245-3248, 1999. 
Scoville, J., Sornette, J., and Freund, F.: Paradox of peroxy defects and positive holes in rocks Part II: Outflow of electric currents from stressed rocks, J. Asian Earth Sci., 114, 338-351, 2015.

Simpson, J. J. and Taflove, A.: Electrokinetic effect of the Loma Prieta earthquake calculated by an entire-Earth FDTD solution of Maxwell's equations, Geophys. Res. Lett., 32, L09302, doi:10.1029/2005GL022601, 2005.

Sorokin, V. M. and Hayakawa, M.: Generation of Seismic-Related DC Electric Fields and Lithosphere-Atmosphere-Ionosphere Coupling, Modern Appl. Sci., 7, 1-25, 2013.

Sorokin, V. M. and Hayakawa, M.: Plasma and Electromagnetic Effects Caused by the Seismic-Related Disturbances of Electric Current in the Global Circuit, Modern Appl. Sci., 8, 61-83, 2014.

Uyeda, S., Nagao, T., Orihara, Y., Yamaguchi, T., and Takahashi I.: Geoelectric potential changes: Possible precursors to earthquakes in Japan, P. Natl. Acad. Sci. USA, 97, 4561-4566, 2000.

Varotsos, P. and Lazaridou, M.: Latest aspects of earthquake prediction in Greece based on seismic electric signals, Tectonophysics, 188, 321-347, 1991.

Varotsos, P., Sarlis, N., and Lazaridou, M.: Transmission of stress induced electric signals in dielectric media, Part II, Acta Geophys., 48, 141-177, 2000.

Varotsos, P., Sarlis, N., Skordas, E., Tanaka, H., and Lazaridou, M.: Additional evidence on some relationship between seismic electric signals and earthquake source parameters, Acta Geophys., 53, 293-298, 2005.

Wait, J. R.: Geo-electromagnetism: Academic Press, 1982.

Wait, J. R.: Some Factors Concerning Electromagnetic Wave Propagation in the Earth's Crust, Proc. IEEE, 54, August 1966.

$\mathrm{Xu}$, C., Di, Q. Y., Fu, C. M., and Wang, M. Y.: The contrast of response characteristics between large power long dipole and circle source, Chinese J. Geophys., 55, 2097-2104, doi:10.6038/j.issn.0001-5733.2012.06.03, 2012 (in Chinese with English abstract)

$\mathrm{Xu}, \mathrm{T}$., Hu, Y., Wu, J., Wu, Z., Suo, Y., and Feng, J.: Giant disturbance in the ionospheric F2 region prior to the M8.0 Wenchuan earthquake on 12 May 2008, Ann. Geophys., 28, 1533-1538, 2010 .
Xu, X. W.: Album of 5.12 Wenchuan 8.0 earthquake surface ruptures, Seismological press, 2009 (in Chinese with English abstract).

Yu, T., Mao, T., Wang, Y. G., and Wang, J. S.: Study of the ionospheric anomaly before the Wenchuan earthquake, Chinese Sci. Bull., 54, 1086-1092, doi:10.1007/s11434-008-0587-8, 2009 (in Chinese with English abstract).

Zeng, Z. C., Zhang, B., Fang, G. Y., Wang, D. F., and Yin, H. J.: The analysis of ionospheric variations before Wenchuan earthquake with DEMETER data, Chinese J. Geophys., 52, 11-19, 2009 (in Chinese with English abstract).

Zhang, Y., Feng, W. P., Xu, L. S., Zhou, C. H., and Chen, Y. T.: Spatio-temporal rupture process of the 2008 great Wenchuan earthquake, Science China Ser. D, 52, 145-154, 2009a.

Zhang, X., Shen, X., Liu, J., Ouyang, X., Qian, J., and Zhao, S.: Analysis of ionospheric plasma perturbations before Wenchuan earthquake, Nat. Hazards Earth Syst. Sci., 9, 1259-1266, doi:10.5194/nhess-9-1259-2009, 2009b.

Zhuang, J., Vere-Jones, D., Guan, H., Ogata, Y., and Ma, L.: Preliminary analysis of observations on the ultra-low frequency electric field in the Beijing region, Pure Appl. Geophys., 162, 13671396, 2005.

Zhu, Y. T., Wang, X. B., Yu, N., Gao, S. Q., Li, K., and Shi, Y. J.: Longmenshan magnetotelluric deep structure and the Wenchuan earthquake $\left(M_{S} 8.0\right)$, Acta Geologica Sinica, 82, 1769-777, 2008 (in Chinese with English abstract).

Zolotov, O. V.: Ionosphere Quasistatic Electric Fields Disturbances over Seismically Active Regions as Inferred from SatelliteBased Observations: A Review, Russian Journal of Physical Chemistry B, 9, 85-788, 2015.

Zolotov, O. V., Namgaladze, A. A., Zakharenkova, I. E., Martynenko, O. V., and Shagimuratov, I. I.: Physical Interpretation and Mathematical Simulation of Ionospheric Precursors of Earthquakes at Midlatitudes, Geomagnetism Aeronomy, 52, 390-397, 2012. 